\title{
Treading a Fine Line: Characterisations and Impossibilities for Liberal Principles in Infinitely-Lived Societies ${ }^{1}$
}

\author{
Michele Lombardi ${ }^{2} \quad$ Roberto Veneziani ${ }^{3}$
}

March 14, 2012

\footnotetext{
${ }^{1}$ We are grateful to José Carlos Rodriguez Alcantud, Geir Asheim, Kaushik Basu, Bhaskar Dutta, Peter Hammond, Sushama Murty, Koichi Tadenuma, Naoki Yoshihara, and audiences at Hitotsubashi University, the University of Warwick (CRETA), K.U. Leuven, the University of Maastricht, the University of Massachusetts (Amherst), the Midwest Political Science Association conference (Chicago), and the New Directions in Welfare conference (Oxford) for insightful comments and suggestions. Special thanks go to Marco Mariotti for many enlightening discussions. The usual disclaimer applies.

${ }^{2}$ Department of Quantitative Economics, Maastricht University, P.O. Box 616, NL-6200 MD Maastricht, Netherlands, phone: 003143388 3761, fax: 0031 43388 2000, e-mail: m.lombardi@maastrichtuniversity.nl. Department of Economics, University of Surrey, GU2 7XH Guildford, Surrey, United Kingdom, e-mail: m.lombardi@surrey.ac.uk.

${ }^{3}$ School of Economics and Finance, Queen Mary University of London, London, E1 4NS, United Kingdom, phone: 0044207882 8852, fax: 0044208983 3580, e-mail: r.veneziani@qmul.ac.uk
} 


\begin{abstract}
This paper extends the analysis of liberal principles in social choice recently proposed by Mariotti and Veneziani (2009a) to infinitely-lived societies. First, some novel characterisations of inegalitarian leximax social welfare relations are derived based on the Individual Benefit Principle (IBP), which incorporates a liberal, non-interfering view of society. This is surprising because the IBP has no obvious inegalitarian content. Second, some impossibility results are derived that highlight a general tension between standard fairness and efficiency axioms in social choice, and a liberal Principle of Non-Interference that generalises IBP.

JEL classification: D63; D70.
\end{abstract}

Key-words: Infinite utility streams, Individual Benefit Principle, leximax, Non-Interference, impossibility. 


\section{Introduction}

Liberal principles in philosophy and social choice tend to express some notion of individual autonomy or freedom. In a recent contribution, Mariotti and Veneziani (2009a) have proposed a new axiom - called the Harm Principle (HP) - suited for Social Welfare Orderings (swos), which captures a liberal view of non-interference inspired by J.S. Mill's conception of liberty. The basic content of HP can be illustrated as follows: consider two welfare allocations $u$ and $v$ such that $u$ is socially preferred to $v$, and two different allocations $u^{\prime}$ and $v^{\prime}$ such that agent $i$ is worse off at these than at the corresponding starting allocations, the other agents are equally well off, and agent $i$ prefers $u^{\prime}$ to $v^{\prime}$. Whatever the cause of the decrease in agent $i$ 's welfare, HP requires that society's preferences over $u^{\prime}$ and $v^{\prime}$ agree with person $i$ 's preferences: having already suffered a welfare loss in both allocations, and given that nobody else is affected, agent $i$ should not be punished in the swo by changing social preferences against her.

Although HP incorporates no egalitarian content, Mariotti and Veneziani (2009a) have shown that, together with the standard axioms of Anonymity and Strong Pareto, it characterises the leximin SWO in societies with a finite number of agents. Lombardi and Veneziani (2009) have generalised this surprising result by weakening $\mathbf{H P}$ and, based on the weak HP, they have provided novel characterisations of various Swos related to Rawls's difference principle. They have also used the weak $\mathbf{H P}$ to characterise the leximin social welfare relations (swRs) proposed by Asheim and Tungodden (2004) and by Bossert et al. (2007) in infinitely-lived economies. The latter results are particularly interesting because the analysis of infinitely-lived societies is crucial, especially in the discussion of intergenerational justice, and the definition of suitable criteria to evaluate allocations is still an open question in the infinite context (among recent contributions see, for example, Lauwers, 1997; Basu and Mitra, 2003; Zame 2007; Hara et al., 2008; Crespo et al., 2009; Alcantud and Garcìa-Sanz, 2010; Lauwers, 2010. For a thorough 
review, see Asheim, 2010).

This paper extends the analysis of liberal, non-interfering views in infinitelylived societies in two main directions. First, the Individual Benefit Principle (IBP) - proposed by Mariotti and Veneziani (2010) in economies with a finite number of agents - is analysed. The IBP also incorporates a liberal, non-interfering view of society and it can be taken as the theoretical complement of HP, for it requires society not to switch its preferences when agent $i$ 's welfare in both allocations $u$ and $v$ above increases. It is shown that a weaker version of IBP suitable for SWRs in infinitely-lived societies, together with some standard axioms, characterises various inegalitarian leximax swRs. These results are interesting per se, because the IBP has no obvious inegalitarian content, and because they provide the first characterisations of leximax SWRs in the infinite context. But they also have relevant implications for liberal approaches to social choice.

In fact, second, as argued by Mariotti and Veneziani (2009b, 2010), HP and IBP should be seen as two parts of a single liberal principle of NonInterference. In finite economies, Mariotti and Veneziani (2010) prove that any swo that satisfies the principle of Non-Interference and Weak Pareto Optimality must be dictatorial. This arguably raises serious questions on liberal, non-interfering approaches in social choice and political philosophy. In this paper, a weak principle of Non-Interference suited for swRs in infinitelylived societies is proposed, and two impossibility results are proved which confirm the existence of a general conflict between liberal principles of noninterference and standard fairness and efficiency axioms. This conflict arises even if no requirements of continuity are imposed and it derives from the general tension between two conflicting SwRs that are simultaneously implied by the axioms. 


\section{The Framework}

Let $X \equiv \mathbb{R}^{\mathbb{N}}$ be the set of countably infinite utility streams, where $\mathbb{R}$ is the set of real numbers and $\mathbb{N}$ is the set of natural numbers. An element of $X$ is ${ }_{1} u=\left(u_{1}, u_{2}, \ldots\right)$ and $u_{t}$ is the utility of a representative member of generation $t \in \mathbb{N}$. For any $T \in \mathbb{N}$ and any ${ }_{1} u \in X,{ }_{1} u_{T}=\left(u_{1}, \ldots, u_{T}\right)$ denotes the $T$-head of ${ }_{1} u$ and ${ }_{T+1} u=\left(u_{T+1}, u_{T+2}, \ldots\right)$ denotes its $T$-tail, so that the stream ${ }_{1} u$ can be written as ${ }_{1} u=\left({ }_{1} u_{T},{ }_{T+1} u\right)$.

A permutation $\pi$ is a bijective mapping of $\mathbb{N}$ onto itself. A permutation $\pi$ of $\mathbb{N}$ is finite whenever there is $T \in \mathbb{N}$ such that $\pi(t)=t, \forall t>T$. Let $\Pi$ be the set of all finite permutations. For any ${ }_{1} u \in X$ and any permutation $\pi$, let $\pi\left({ }_{1} u\right)=\left(u_{\pi(t)}\right)_{t \in \mathbb{N}}$ be a permutation of ${ }_{1} u$. For any $T \in \mathbb{N}$ and ${ }_{1} u \in X,{ }_{1} \bar{u}_{T}$ is a permutation of ${ }_{1} u_{T}$ such that the components are ranked in ascending order.

For any ${ }_{1} u,{ }_{1} v \in X$, we write ${ }_{1} u \geq{ }_{1} v$ to mean $u_{t} \geq v_{t}, \forall t \in \mathbb{N} ;{ }_{1} u>{ }_{1} v$ to mean ${ }_{1} u \geq{ }_{1} v$ and ${ }_{1} u \neq{ }_{1} v$; and ${ }_{1} u \gg{ }_{1} v$ to mean $u_{t}>v_{t}, \forall t \in \mathbb{N}$.

Let $\succcurlyeq$ be a binary relation over $X$. For any ${ }_{1} u,{ }_{1} v \in X$, we write ${ }_{1} u \succcurlyeq{ }_{1} v$ for $\left({ }_{1} u,{ }_{1} v\right) \in \succcurlyeq$ and ${ }_{1} u \nsucc{ }_{1} v$ for $\left({ }_{1} u,{ }_{1} v\right) \notin \succcurlyeq ; \succcurlyeq$ stands for "at least as good as". For any ${ }_{1} u,{ }_{1} v \in X$, the asymmetric factor $\succ$ of $\succcurlyeq$ is defined by ${ }_{1} u \succ{ }_{1} v$ if and only if ${ }_{1} u \succcurlyeq{ }_{1} v$ and ${ }_{1} v \nsucc{ }_{1} u$, and the symmetric part $\sim$ of $\succcurlyeq$ is defined by ${ }_{1} u \sim{ }_{1} v$ if and only if ${ }_{1} u \succcurlyeq{ }_{1} v$ and ${ }_{1} v \succcurlyeq{ }_{1} u$. Throughout the paper, $\succcurlyeq$ on $X$ is assumed to be reflexive and transitive, but not necessarily complete. Let $\succcurlyeq$ and $\succcurlyeq^{\prime}$ be relations on $X, \succcurlyeq^{\prime}$ is an extension of $\succcurlyeq$ if $\succcurlyeq \subseteq \succcurlyeq^{\prime}$ and $\succ \subseteq \succ^{\prime}$.

\section{The Harm Principle}

A standard definition of the leximin swR used in the literature to compare countably infinite utility streams is due to Asheim and Tungodden (2004; Definition 2, p. 224). This definition can be stated as follows:

Definition 1. $\succcurlyeq^{L M}$ is the leximin SWR if, $\forall_{1} u,{ }_{1} v \in X$ :

(i) ${ }_{1} u \sim^{L M}{ }_{1} v \Leftrightarrow \exists \tilde{T} \geq 1$ such that $\forall T \geq \tilde{T}:{ }_{1} \bar{u}_{T}={ }_{1} \bar{v}_{T}$; and, 
(ii) ${ }_{1} u \succ^{L M}{ }_{1} v \Leftrightarrow \exists \tilde{T} \geq 1$ such that, $\forall T \geq \tilde{T}, \exists t \in\{1, \ldots, T\}$ with $\bar{u}_{s}=$ $\bar{v}_{s}(\forall 1 \leq s<t)$ and $\bar{u}_{t}>\bar{v}_{t}$.

The characterisation of the leximin SWR $\succcurlyeq^{L M}$ by Lombardi and Veneziani (2009) is based on the following axioms.

Finite Anonymity, FA: $\forall{ }_{1} u \in X$ and $\forall \pi \in \Pi, \pi\left({ }_{1} u\right) \sim{ }_{1} u$.

Strong Pareto Optimality, SPO: $\forall_{1} u,{ }_{1} v \in X:{ }_{1} u>{ }_{1} v \Rightarrow{ }_{1} u \succ_{1} v$.

FA and SPO incorporate standard principles of fairness and efficiency, respectively, and need no further comment.

Weak Preference Continuity, WPC: $\forall_{1} u,{ }_{1} v \in X: \exists \tilde{T} \geq 1$ such that $\left({ }_{1} u_{T},{ }_{T+1} v\right) \succ{ }_{1} v \forall T \geq \tilde{T} \Rightarrow{ }_{1} u \succ{ }_{1} v$.

WPC has been proposed by Asheim and Tungodden (2004): it establishes "a link to the standard finite setting of distributive justice, by transforming the comparison of any two infinite utility paths to an infinite number of comparisons of utility paths each containing a finite number of generations" (Asheim and Tungodden, 2004; p. 223). ${ }^{1}$

Minimal Completeness, MC: $\forall_{1} u,{ }_{1} v \in X, \exists T \geq 1\left({ }_{1} u_{T},{ }_{T+1} v\right) \neq{ }_{1} v$ $\Rightarrow\left({ }_{1} u_{T}, T+1 v\right) \succcurlyeq{ }_{1} v$ or ${ }_{1} v \succcurlyeq\left({ }_{1} u_{T},{ }_{T+1} v\right)$.

MC states that a SWR should be able to compare (at least) vectors with the same tail: this seems an obviously desirable property, as it imposes a minimum requirement of completeness.

Weak Harm Principle, HP: $\forall_{1} u,{ }_{1} v,{ }_{1} u^{\prime},{ }_{1} v^{\prime} \in X: \exists T \geq 1{ }_{1} u=$ $\left({ }_{1} u_{T},{ }_{T+1} v\right) \succ{ }_{1} v$, and ${ }_{1} u^{\prime},{ }_{1} v^{\prime}$ are such that, $\exists i \leq T$,

\footnotetext{
${ }^{1}$ Although WPC may be seen as a purely technical requirement to deal with infinitedimensional vectors, it does entail a preference for earlier generations, owing to its reliance on a natural order. As argued below, none of the key insights of this paper depends on a natural ordering of generations. We thank Geir Asheim for alerting us to this issue.
} 


$$
\begin{aligned}
u_{i}^{\prime} & <u_{i}, \\
v_{i}^{\prime} & <v_{i}, \\
u_{j}^{\prime} & =u_{j}, \forall j \neq i, \\
v_{j}^{\prime} & =v_{j}, \forall j \neq i,
\end{aligned}
$$

implies ${ }_{1} v^{\prime} \nsucc{ }_{1} u^{\prime}$ whenever $u_{i}^{\prime}>v_{i}^{\prime}$.

HP formalises the Harm Principle in infinitely-lived societies. It is weaker than the version analysed by Mariotti and Veneziani (2009a), because it does not require that ${ }_{1} u^{\prime} \succ{ }_{1} v^{\prime}$ and instead it emphasises the negative prescription of the Harm Principle by simply ruling out the reversal of strict preferences. Moreover it only holds for vectors with the same tail. ${ }^{2}$

Based on these axioms, Lombardi and Veneziani (2009; Theorem 3) prove the following result.

Theorem 1. $\succcurlyeq$ is an extension of $\succcurlyeq^{L M}$ if and only if $\succcurlyeq$ satisfies FA, SPO, HP, WPC, and MC.

As Mariotti and Veneziani (2009a) have argued, a characterisation of the leximin based on HP is surprising, because $\mathbf{H P}$ has no obvious egalitarian content, unlike the standard axiom of Hammond Equity (see, e.g., Hammond, 1976; Asheim and Tungodden, 2004). Equally surprising is that, by a germane perturbation of $\mathbf{H P}$, it is possible to characterise the strongly inegalitarian leximax SWR.

\section{The Benefit Principle and Non-Interference}

According to the leximax, that society is best which (lexicographically) maximises the welfare of its best-off members. In infinitely-lived economies, this

\footnotetext{
${ }^{2}$ The philosophical foundations of $\mathbf{H P}$ (and of the principle of Non-Interference introduced below) are extensively analysed in Mariotti and Veneziani (2009b), in relation to classical liberal political philosophy. For a more detailed discussion of the axioms, see also Lombardi and Veneziani (2009).
} 
intuition can be formalised as follows.

Definition 2. $\succcurlyeq^{L X}$ is the leximax SWR if, $\forall_{1} u,{ }_{1} v \in X$ :

(i) ${ }_{1} u \sim^{L X}{ }_{1} v \Leftrightarrow \exists \tilde{T} \geq 1$ such that $\forall T \geq \tilde{T}:{ }_{1} \bar{u}_{T}={ }_{1} \bar{v}_{T}$; and,

(ii) ${ }_{1} u \succ^{L X}{ }_{1} v \Leftrightarrow \exists \tilde{T} \geq 1$ such that, $\forall T \geq \tilde{T}, \exists t \in\{1, \ldots, T\}$ with $\bar{u}_{s}=$ $\bar{v}_{s}(\forall t<s \leq T)$ and $\bar{u}_{t}>\bar{v}_{t}$.

In order to characterise the leximax SWR, the same axioms as for the leximin are used, except for $\mathbf{H P}$, which is substituted with the Individual Benefit Principle. The IBP also captures a liberal requirement of noninterference and can be formalised as follows.

Individual Benefit PRInciple, IBP: $\forall_{1} u,{ }_{1} v,_{1} u^{\prime},_{1} v^{\prime} \in X: \exists T \geq 1_{1} u=$ $\left({ }_{1} u_{T}, T+1=\right) \succ{ }_{1} v$, and ${ }_{1} u^{\prime},{ }_{1} v^{\prime}$ are such that, $\exists i \leq T$,

$$
\begin{aligned}
u_{i}^{\prime} & >u_{i}, \\
v_{i}^{\prime} & >v_{i}, \\
u_{j}^{\prime} & =u_{j}, \forall j \neq i, \\
v_{j}^{\prime} & =v_{j}, \forall j \neq i,
\end{aligned}
$$

implies ${ }_{1} v^{\prime} \nsucc{ }_{1} u^{\prime}$ whenever $u_{i}^{\prime}>v_{i}^{\prime}$.

IBP stipulates that after an increase in welfare that concerns only agent $i$, society should not reverse the strict preference between ${ }_{1} u$ and ${ }_{1} v$ to a strict preference for ${ }_{1} v^{\prime}$ over ${ }_{1} u^{\prime}$, possibly except when $i$ prefers otherwise. Whatever the cause of the increase in welfare for agent $i$, society should not 'punish' $i$ by reversing its strict preferences, given that nobody else is affected and therefore for all other agents the choice between ${ }_{1} u^{\prime}$ and ${ }_{1} v^{\prime}$ is the same as between ${ }_{1} u$ and ${ }_{1} v$.

The liberal moral intuition behind IBP is similar to the $\mathbf{H P}$, and yet Theorem 2 proves that IBP leads to a rather different result.

Theorem 2. $\succcurlyeq$ is an extension of $\succcurlyeq^{L X}$ if and only if $\succcurlyeq$ satisfies FA, SPO, IBP, WPC, and MC. 
Proof. $(\Rightarrow)$ Let $\succcurlyeq^{L X} \subseteq \succcurlyeq$. It is easy to see that $\succcurlyeq$ meets FA, SPO, WPC, and MC. We show that $\succcurlyeq$ satisfies IBP. Take any ${ }_{1} u,{ }_{1} v,{ }_{1} u^{\prime},{ }_{1} v^{\prime} \in X$ such that ${ }_{1} u=\left({ }_{1} u_{T},{ }_{T+1} v\right) \succ{ }_{1} v \exists T \geq 1$, and ${ }_{1} u^{\prime},{ }_{1} v^{\prime}$ are such that, $\exists i \leq T$, $u_{i}^{\prime}>u_{i}, v_{i}^{\prime}>v_{i}, u_{j}^{\prime}=u_{j} \forall j \neq i, v_{j}^{\prime}=v_{j} \forall j \neq i$. We show that ${ }_{1} u^{\prime} \succ_{1} v^{\prime}$ whenever $u_{i}^{\prime}>v_{i}^{\prime}$. As ${ }_{1} u,{ }_{1} v$ have the same tail, ${ }_{1} u \succ{ }^{L X}{ }_{1} v$. Then, $\exists \tilde{T} \geq 1$ such that $\forall T^{\prime} \geq \tilde{T}$ there is a $t \in\left\{1, \ldots, T^{\prime}\right\}$ such that $\bar{u}_{s}=\bar{v}_{s} \forall t<s \leq T^{\prime}$, and $\bar{u}_{t}>\bar{v}_{t}$. Take any $T^{\prime} \geq \tilde{T}$. If $\bar{u}_{T^{\prime}}>\bar{v}_{T^{\prime}}$, then the result follows since $\bar{u}_{T^{\prime}}^{\prime} \in\left\{u_{i}^{\prime}, \bar{u}_{T^{\prime}}\right\}$ and $\bar{v}_{T^{\prime}}^{\prime} \in\left\{v_{i}^{\prime}, \bar{v}_{T^{\prime}}\right\}$. Otherwise, let $\bar{u}_{T^{\prime}}=\bar{v}_{T^{\prime}}$. The result is readily obtained if $\bar{v}_{k}=\bar{v}_{k}^{\prime} \forall t \leq k \leq T^{\prime}$. Thus, let $\bar{v}_{k} \neq \bar{v}_{k}^{\prime}$ for some $t \leq k \leq T^{\prime}$. We proceed according to whether $\bar{v}_{t}<v_{i}^{\prime}<\bar{v}_{t+1}$ or not.

Case 1. $\bar{v}_{t}<v_{i}^{\prime}<\bar{v}_{t+1}$

Then, $\bar{v}_{t+1}^{\prime}>\bar{v}_{t}^{\prime}=v_{i}^{\prime}>\bar{v}_{t}$ and $\bar{v}_{s}^{\prime}=\bar{v}_{s} \forall T^{\prime} \geq s>t$. If $u_{i}^{\prime} \in\left(v_{i}^{\prime}, \bar{u}_{t+1}\right]$, then $\bar{u}_{t}^{\prime}>v_{i}^{\prime}=\bar{v}_{t}^{\prime}$. Otherwise, let $u_{i}^{\prime}>\bar{u}_{t+1}$. Thus, there exists $j \geq t+1$ such that $u_{i}^{\prime}=\bar{u}_{j}^{\prime}>\bar{u}_{j}$. Let

$$
m \equiv \max \left\{t+1 \leq j \leq T^{\prime} \mid \bar{u}_{j}^{\prime}>\bar{u}_{j}\right\} .
$$

Since $\bar{v}_{j}^{\prime}=\bar{v}_{j}=\bar{u}_{j} \forall t<j \leq T^{\prime}$ it follows that $\bar{u}_{m}^{\prime}>\bar{v}_{m}^{\prime}$. In either case, we conclude that there exists $t^{*} \leq T^{\prime}$ such that $\bar{u}_{s}^{\prime}=\bar{v}_{s}^{\prime} \forall t^{*}<s \leq T^{\prime}$ and $\bar{u}_{t^{*}}^{\prime}>\bar{v}_{t^{*}}^{\prime}$, as sought.

Case 2. $v_{i}^{\prime} \geq \bar{v}_{t+1}$

If $v_{i}^{\prime} \geq \bar{v}_{T^{\prime}}$, then $\bar{u}_{T^{\prime}}^{\prime}>\bar{v}_{T^{\prime}}^{\prime}$ as $u_{i}^{\prime}>v_{i}^{\prime}$. Otherwise, let $v_{i}^{\prime}<\bar{v}_{T^{\prime}}$. Let

$$
\ell \equiv \min \left\{t+1<j \leq T^{\prime} \mid v_{i}^{\prime}<\bar{v}_{j}\right\} .
$$

Then, $\bar{v}_{\ell}>v_{i}^{\prime}=\bar{v}_{\ell-1}^{\prime} \geq \bar{u}_{\ell-1}=\bar{v}_{\ell-1}$. Since $v_{i}^{\prime}<u_{i}^{\prime}$, then $u_{i}^{\prime}>\bar{u}_{\ell-1}$. If $u_{i}^{\prime} \in\left(\bar{u}_{\ell-1}, \bar{u}_{\ell}\right]$, then $\bar{v}_{\ell-1}^{\prime}<u_{i}^{\prime}=\bar{u}_{\ell-1}^{\prime}$. Otherwise, let $\bar{u}_{\ell}<u_{i}^{\prime}$. Then, there exists $\ell<m \leq T^{\prime}$ such that $\bar{v}_{m}^{\prime}=\bar{v}_{m}<\bar{u}_{m}^{\prime}=u_{i}^{\prime}$. Furthermore, $\bar{u}_{s}^{\prime}=$ $\bar{v}_{s}^{\prime} \forall m<s \leq T^{\prime}$ if $m<T^{\prime}$. In either case, we have found a $t^{*} \in\left\{t, \ldots, T^{\prime}\right\}$ such that $\bar{u}_{s}^{\prime}=\bar{v}_{s}^{\prime} \forall t^{*}<s \leq T^{\prime}$ and $\bar{u}_{t^{*}}^{\prime}>\bar{v}_{t^{*}}^{\prime}$.

Since the above arguments hold for any $T^{\prime} \geq \tilde{T}$, and by our supposition $\succcurlyeq^{L X} \subseteq \succcurlyeq$, we have that ${ }_{1} u^{\prime} \succ{ }_{1} v^{\prime}$, as sought. 
$(\Leftarrow)$ Suppose that $\succcurlyeq$ satisfies FA, SPO, IBP, WPC, and MC. We show that $\sim^{L X} \subseteq \sim$ and $\succ^{L X} \subseteq \succ$. Take any ${ }_{1} u,{ }_{1} v \in X$.

If ${ }_{1} u \sim^{L X}{ }_{1} v$, then by definition, $\exists \tilde{T} \geq 1$ such that $\forall T \geq \tilde{T}:{ }_{1} \bar{u}_{T}={ }_{1} \bar{v}_{T}$, and so ${ }_{T+1} u={ }_{T+1} v \forall T>\tilde{T}$. By FA it follows that ${ }_{1} u \sim{ }_{1} v$, as sought.

Next, suppose that ${ }_{1} u \succ^{L X}{ }_{1} v$. By definition $\exists \tilde{T} \geq 1$ such that, $\forall T \geq \tilde{T}$, $\exists t \in\{1, \ldots, T\}$ with $\bar{u}_{s}=\bar{v}_{s}(\forall t<s \leq T)$ and $\bar{u}_{t}>\bar{v}_{t}$. Take any $T \geq \tilde{T}$ and consider the vector ${ }_{1} w \equiv\left({ }_{1} u_{T},{ }_{T+1} v\right)$. Note that ${ }_{1} w \succ^{L X}{ }_{1} v$. By contradiction, we prove that ${ }_{1} w \succ{ }_{1} v$. By FA and transitivity, we can consider ${ }_{1} \bar{w} \equiv\left({ }_{1} \bar{u}_{T},{ }_{T+1} v\right)$ and ${ }_{1} \bar{v} \equiv\left({ }_{1} \bar{v}_{T}, T+1=\right)$. We distinguish two cases. Case 1. Suppose that ${ }_{1} \bar{v} \succ{ }_{1} \bar{w}$.

Then, $\bar{v}_{l}>\bar{w}_{l}, \exists l<t \leq T$, otherwise $\mathbf{S P O}$ yields a contradiction. Let

$$
k \equiv \max \left\{1 \leq l<t \mid \bar{v}_{l}>\bar{w}_{l}\right\}
$$

By FA, let $w_{i}=\bar{w}_{k}$ and $v_{i}=\bar{v}_{k+g} \exists g \in \mathbb{N}$ such that $0<g \leq t-k$ and $\bar{w}_{k+g}>\bar{v}_{k+g}$. Let $d_{1}, d_{2}>0$, and consider vectors ${ }_{1} w^{\prime},{ }_{1} v^{\prime}$ formed from ${ }_{1} \bar{w},{ }_{1} \bar{v}$ as follows: $\bar{v}_{k+g}$ is raised to $\bar{v}_{k+g}+d_{1}$ such that $\bar{w}_{k+g}>\bar{v}_{k+g}+d_{1}$; $\bar{w}_{k}$ is raised to $\bar{w}_{k}+d_{2}$ such that $\bar{v}_{k+g}+d_{1}>\bar{w}_{k}+d_{2}>\bar{v}_{k}$; and all other entries of ${ }_{1} \bar{w}$ and ${ }_{1} \bar{v}$ are unchanged. By FA, consider ${ }_{1} \bar{w}^{\prime}=\left({ }_{1} \bar{w}_{T}^{\prime}, T+1 v\right)$ and ${ }_{1} \bar{v}^{\prime}=\left({ }_{1} \bar{v}_{T}^{\prime}, T+1 v\right)$. By construction $\bar{w}_{j}^{\prime} \geq \bar{v}_{j}^{\prime} \forall j \geq k$, with $\bar{w}_{k+g}^{\prime}>\bar{v}_{k+g}^{\prime}$. IBP implies ${ }_{1} \bar{w}^{\prime} \nsucc{ }_{1} \bar{v}^{\prime}$, and so ${ }_{1} \bar{v}^{\prime} \succcurlyeq{ }_{1} \bar{w}^{\prime}$, by MC. Moreover, by SPO, $d_{1}, d_{2}$ can be chosen so that ${ }_{1} \bar{v}^{\prime} \succ{ }_{1} \bar{w}^{\prime}$, without loss of generality. Consider two sub-cases:

a) Suppose that $\bar{v}_{k}>\bar{w}_{k}$, but $\bar{w}_{l} \geq \bar{v}_{l} \forall l<k$. It follows that ${ }_{1} \bar{w}^{\prime}>{ }_{1} \bar{v}^{\prime}$, and so SPO implies that ${ }_{1} \bar{w}^{\prime} \succ{ }_{1} \bar{v}^{\prime}$, a contradiction.

b) Suppose that $\bar{v}_{l}>\bar{w}_{l} \exists l<k$. Note that by construction $\bar{v}_{l}^{\prime}=\bar{v}_{l}$ and $\bar{w}_{l}^{\prime}=\bar{w}_{l} \forall l<k$. Then, let

$$
k^{\prime} \equiv \max \left\{1 \leq l<k \mid \bar{v}_{l}^{\prime}>\bar{w}_{l}^{\prime}\right\}
$$

The above argument can be applied to ${ }_{1} \bar{w}^{\prime},{ }_{1} \bar{v}^{\prime}$ to derive vectors ${ }_{1} \bar{w}^{\prime \prime},{ }_{1} \bar{v}^{\prime \prime}$ such that $\bar{w}_{j}^{\prime \prime} \geq \bar{v}_{j}^{\prime \prime} \forall j \geq k^{\prime}$, whereas IBP, combined with MC, FA, and 
SPO imply ${ }_{1} \bar{v}^{\prime \prime} \succ{ }_{1} \bar{w}^{\prime \prime}$. And so on. After a finite number of iterations $q$, two vectors ${ }_{1} \bar{w}^{q},{ }_{1} \bar{v}^{q}$ can be derived such that, by IBP, combined with MC, $\mathbf{F A}$, and SPO, ${ }_{1} \bar{v}^{q} \succ{ }_{1} \bar{w}^{q}$ but, by $\mathbf{S P O},{ }_{1} \bar{w}^{q} \succ{ }_{1} \bar{v}^{q}$, which is a contradiction. Case 2. Suppose that ${ }_{1} \bar{v} \sim{ }_{1} \bar{w}$.

By assumption, $\bar{v}_{t}<\bar{u}_{t} \equiv \bar{w}_{t}$. Therefore, define ${ }_{1} \bar{w}^{\prime}$ as follows: $\bar{w}_{\tau}^{\prime}=\bar{w}_{\tau}$ $\forall \tau \in \mathbb{N} \backslash\{t\}$ and $\bar{w}_{t}^{\prime}=\bar{w}_{t}-\epsilon>\bar{v}_{t}, \exists \epsilon>0$. By SPO and transitivity, it follows that ${ }_{1} \bar{v} \succ{ }_{1} \bar{w}^{\prime}$ but ${ }_{1} \bar{w}^{\prime} \succ^{L X}{ }_{1} \bar{v}$. Hence, the argument of Case 1 above can be applied to ${ }_{1} \bar{v}$ and ${ }_{1} \bar{w}^{\prime}$, which produces the desired contradiction.

Since ${ }_{1} \bar{v} \nsucc{ }_{1} \bar{w}, \mathbf{M C}$ implies ${ }_{1} \bar{w} \succ{ }_{1} \bar{v}$. FA and transitivity imply that $\left({ }_{1} u_{T},{ }_{T+1} v\right) \succ{ }_{1} v$. Since this is true for any $T \geq \tilde{T}$, WPC implies ${ }_{1} u \succ{ }_{1} v$, as sought. ${ }^{3}$

Thus, a seemingly mild liberal principle, together with standard fairness and efficiency axioms, leads straight to a strongly inegalitarian criterion. But Theorem 2 has another interesting theoretical implication. Consider the next axiom, which incorporates the normative intuitions behind HP and IBP in a unified liberal framework, and generalises the principle of Non-Interference proposed by Mariotti and Veneziani (2010) to infinitelylived economies.

Non-Interference, NI: $\forall{ }_{1} u,{ }_{1} v,{ }_{1} u^{\prime},{ }_{1} v^{\prime} \in X: \exists T \geq 1{ }_{1} u=\left({ }_{1} u_{T},{ }_{T+1} v\right) \succ$ ${ }_{1} v$, and ${ }_{1} u^{\prime},{ }_{1} v^{\prime}$ are such that, $\exists i \leq T$,

$$
\begin{aligned}
\left(u_{i}^{\prime}-u_{i}\right)\left(v_{i}^{\prime}-v_{i}\right) & >0, \\
u_{j}^{\prime} & =u_{j}, \forall j \neq i, \\
v_{j}^{\prime} & =v_{j}, \forall j \neq i,
\end{aligned}
$$

implies ${ }_{1} v^{\prime} \nsucc{ }_{1} u^{\prime}$ whenever $u_{i}^{\prime}>v_{i}^{\prime}$.

Axiom NI captures the following liberal intuition: changes in one individual's welfare that leave all other agents unaffected should not be a motive

\footnotetext{
${ }^{3}$ For the sake of completeness of the proof, the axioms are independent (see the Addendum).
} 
for penalising the individual in the social welfare judgement (by switching strict preferences against her), whether the change involves a damage or a benefit for her.

Theorems 1 and 2 reveal a fundamental tension between standard axioms in social choice and liberal principles of non-interference.

Theorem 3. There exists no swr on $X$ that satisfies FA, SPO, WPC, MC, and NI.

Proof. Suppose, contrary to the statement, that there is an swr $\succcurlyeq$ on $X$ that satisfies FA, SPO, WPC, MC, and NI. Let $\nu, \mu \in \mathbb{R}$, with $\nu>\mu$, and consider vectors ${ }_{1} u,{ }_{1} v \in X$ such that $u_{t}=\mu, \forall t$, whereas $v_{1}<\mu$ and $v_{t}=\nu, \forall t \geq 2$. By Theorem 1, ${ }_{1} u \succ{ }_{1} v$, but by Theorem 2, ${ }_{1} v \succ_{1} u$, a contradiction.

Theorem 3 suggests that a fully non-interfering liberal approach does not provide adequate foundations for social welfare judgements in infinitely-lived societies. It is not possible to grant a universal protection from interference to individuals, even in cases where nobody else is affected.

This insight is robust and the basic structure of the above argument can be extended in various directions. The impossibility result, for example, does not crucially depend on the existence of a natural ordering of generations. It is not difficult to characterise a leximin time-invariant overtaking (Asheim et al., 2010; Definition 4, p. 529) by reformulating the anonymity, Pareto, and minimal completeness axioms as well as the Harm Principle on finite subsets of agents independent of a natural ordering, and by replacing WPC with a time-invariant preference continuity axiom (Asheim et al., 2010; p. $524)$ that also does not depend on a natural ordering of agents. The dual characterisation of a leximax time-invariant overtaking is similarly obtained based on a time-invarant version of the Individual Benefit Principle and an impossibility result analogous to Theorem 3 immediately ensues. ${ }^{4}$

\footnotetext{
${ }^{4}$ The proofs of these claims are straightforward modifications of the results in Asheim
} 
Further, in a recent paper, Alcantud (2011) proves that various impossibility results for liberal principles emerge if one focuses on standard axioms of upper, or lower, semicontinuity in the sup topology, instead of WPC.

It is indeed well-known that continuity requirements in the evaluation of infinite utility streams tend to produce impossibility results (see, e.g., the classic paper by Diamond, 1965; for a thorough general analysis, see Hara et al., 2008). It is therefore interesting to develop the analysis of liberal principles of non-interference without imposing any continuity requirements. This is the task of the next section.

\section{$5 \quad$ Liberal Principles Reconsidered}

This section analyses liberal principles of non-interference in the framework proposed by Bossert et al. (2007). To this end, additional notation is needed.

For each $T \in \mathbb{N}$, let $X_{T}$ denote the set of utility streams of $X$ truncated at $T$. The leximax swo on $X_{T}$ is denoted by $\succcurlyeq_{T}^{L X}$. Then, $\forall_{1} u_{T},{ }_{1} v_{T} \in X_{T}$, the asymmetric factor $\succ_{T}^{L X}$ of $\succcurlyeq_{T}^{L X}$ is defined by:

${ }_{1} u_{T} \succ_{T}^{L X}{ }_{1} v_{T} \Leftrightarrow \bar{u}_{T}>\bar{v}_{T}$ or $\left[\exists t \leq T: \bar{u}_{j}=\bar{v}_{j}(\forall j \leq T\right.$, with $t<j)$, and $\left.\bar{u}_{t}>\bar{v}_{t}\right]$,

whereas the symmetric factor $\sim_{T}^{L X}$ of $\succcurlyeq_{T}^{L X}$ by:

$$
{ }_{1} u_{T} \sim{ }_{T}^{L X}{ }_{1} v_{T} \Leftrightarrow{ }_{1} u_{T}=\pi\left({ }_{1} v_{T}\right) \exists \pi \in \Pi .
$$

The leximax catching-up SWR $\succcurlyeq^{L}$ on $X$ can be formulated as follows. Define a relation $\succcurlyeq_{T}^{L} \subseteq X \times X$ by letting, for all ${ }_{1} u,{ }_{1} v \in X$,

$$
{ }_{1} u \succcurlyeq_{T}^{L} \quad{ }_{1} v \Leftrightarrow{ }_{1} u_{T} \succcurlyeq_{T}^{L X}{ }_{1} v_{T} \text { and }{ }_{T+1} u \geq{ }_{T+1} v .
$$

The relation $\succcurlyeq_{T}^{L}$ is reflexive and transitive for all $T \in \mathbb{N}$. Then the leximax SWR on $X$ is $\succcurlyeq^{L}=\bigcup_{T \in \mathbb{N}} \succcurlyeq_{T}^{L}$ : it is reflexive and transitive, but not necessarily et al. (2010; Section 6.2). The details are available from the authors upon request. 
complete. Moreover, following Bossert et al. (2007; equation (14), p. 586), it can be shown that $\succcurlyeq^{L}$ satisfies the following property:

$$
\forall_{1} u,{ }_{1} v \in X: \exists T \in \mathbb{N} \text { such that }{ }_{1} u \succ_{T}^{L}{ }_{1} v \Leftrightarrow{ }_{1} u \succ^{L}{ }_{1} v \text {. }
$$

The next Theorem characterises the set of ordering extensions of $\succcurlyeq^{L} .^{5}$

Theorem 4. $\succcurlyeq$ is an ordering extension of $\succcurlyeq^{L}$ if and only if $\succcurlyeq$ satisfies FA, SPO, and IBP.

Proof. $(\Rightarrow)$ The proof that any ordering extension of $\succcurlyeq^{L}$ satisfies FA and SPO is immediate. We only need to prove that any ordering extension $\succcurlyeq$ of $\succcurlyeq^{L}$ satisfies IBP. Consider any ${ }_{1} u,{ }_{1} v,{ }_{1} u^{\prime},{ }_{1} v^{\prime} \in X$ such that $\exists T \geq 1$ ${ }_{1} u=\left({ }_{1} u_{T}, T+1 v\right) \succ{ }_{1} v$, and ${ }_{1} u^{\prime},{ }_{1} v^{\prime}$ are such that, $\exists i \leq T, u_{i}^{\prime}>u_{i}, v_{i}^{\prime}>v_{i}$, $u_{j}^{\prime}=u_{j} \forall j \neq i, v_{j}^{\prime}=v_{j} \forall j \neq i$. We show that ${ }_{1} u^{\prime} \succ{ }_{1} v^{\prime}$ whenever $u_{i}^{\prime}>$ $v_{i}^{\prime}$. Since $\succcurlyeq_{T}^{L X}$ is complete and ${ }_{T+1} v={ }_{T+1} u$ it cannot be ${ }_{1} v_{T} \succcurlyeq_{T}^{L X}{ }_{1} u_{T}$, otherwise $\left({ }_{1} v,{ }_{1} u\right) \in \succcurlyeq^{L} \subseteq \succcurlyeq$ which contradicts ${ }_{1} u \succ{ }_{1} v$. Thus, we have that ${ }_{1} u_{T} \succcurlyeq_{T}^{L X}{ }_{1} v_{T},{ }_{1} v_{T} \Varangle_{T}^{L X}{ }_{1} u_{T}$, and ${ }_{T+1} v={ }_{T+1} u$, so that $\left({ }_{1} u,{ }_{1} v\right) \in \succ_{T}^{L}$ by $(1)$. It follows from $(2)$ that $\left({ }_{1} u,{ }_{1} v\right) \in \succ^{L}$. As ${ }_{1} u^{\prime}$ and ${ }_{1} v^{\prime}$ are such that, $\exists i \leq T$, $u_{i}^{\prime}>u_{i}, v_{i}^{\prime}>v_{i}, u_{j}^{\prime}=u_{j} \forall j \neq i, v_{j}^{\prime}=v_{j} \forall j \neq i$, it can easily be shown, as in Mariotti and Veneziani (2010), that ${ }_{1} u_{T}^{\prime} \succ_{T}^{L X}{ }_{1} v_{T}^{\prime}$ whenever $u_{i}^{\prime}>v_{i}^{\prime}$. As ${ }_{T+1} v^{\prime}={ }_{T+1} u^{\prime}$ and ${ }_{1} u_{T}^{\prime} \succ_{T}^{L X}{ }_{1} v_{T}^{\prime}$, it follows from (1) that ${ }_{1} u^{\prime} \succ_{T}^{L}{ }_{1} v^{\prime}$, and therefore ${ }_{1} u^{\prime} \succ^{L}{ }_{1} v^{\prime}$ by (2). But since $\succcurlyeq$ is an ordering extension of $\succcurlyeq^{L}$ it follows that ${ }_{1} u^{\prime} \succ{ }_{1} v^{\prime}$.

$(\Leftarrow)$ The proof is a straightforward modification of the argument in Bossert et al. (2007; Theorem 2, p. 587), using the characterisation of the $T$-person leximax in Mariotti and Veneziani (2010; Proposition 3). ${ }^{6}$

Lombardi and Veneziani (2009; Theorem 5) characterise the set of ordering extensions of the dual leximin catching-up criterion (Bossert et al.,

\footnotetext{
${ }^{5}$ The set of ordering extensions characterised in Theorem 4 is of course non-empty by Szpilrajn's extension theorem.

${ }^{6}$ For the sake of completeness of the proof, the axioms are independent (see the Addendum).
} 
2007; p. 586) using FA, SPO, and HP. Therefore, using a similar argument as in Theorem 3, it immediately follows:

Theorem 5. There exists no SWO on $X$ that satisfies FA, SPO, and NI.

In other words, an impossibility for liberal principles of non-interference ensues even without assuming continuity, provided MC is strengthened to focus on swos.

Theorems 3 and 5 have some interesting theoretical implications. Mariotti and Veneziani (2010) prove that in the finite context any swo that satisfies the principle of non-interference and Weak Pareto Optimality must be dictatorial. Theorems 3 and 5 demonstrate that a general conflict between liberal principles of non-interference and standard axioms of fairness and efficiency exists also in the infinite context. Indeed, the method of proof adopted in this paper forcefully suggests that the incompatibility between the axioms is not due to their clashing in peculiar configurations of welfare levels, but rather to the general tension between two conflicting swRs that are simultaneously implied by the axioms.

In a recent paper, Alcantud (2011) shows that, in the infinite context, the conflict can be mitigated, and possibility results for liberal, non-interfering SWos do emerge, either if SPO is weakened to Weak Pareto Optimality and Monotonicity, and FA is dropped (Alcantud, 2011; Theorem 1 ) ${ }^{7}$ or if FA is upheld at the cost of a further reduction in sensitivity to the welfare of generations by restricting Weak Pareto Optimality to hold only for vectors that become eventually constant (Alcantud, 2011; Remark 2). This suggests that Theorem 5 above and Theorem 1 in Alcantud (2011) can be interpreted as identifying the boundaries of possibility and impossibility results for liberal, non-interfering approaches to intergenerational justice in the infinite context.

\footnotetext{
${ }^{7}$ Equity concerns can be captured by axioms imposing Non-dictatorship of the Present and Non-dictatorship of the Future (Alcantud, 2011; p. 6).
} 


\section{Conclusions}

This paper analyses liberal axioms for swRs in infinitely-lived economies. Various leximax SWRs are characterised by appealing to the Individual Benefit Principle, which incorporates a liberal, non-interfering view of society. This result is interesting per se, since the IBP has no obvious inegalitarian content, and because it provides the first characterisations of the leximax in infinitely-lived economies. It also has relevant implications for liberal approaches to social choice. For it allows us to show that there exists a general tension between standard fairness and efficiency axioms, such as Finite Anonymity and Strong Pareto, and a liberal principle of Non-Interference that generalises IBP.

\section{References}

[1] Alcantud, J.C.R., 2011. Liberal approaches to ranking infinite utility streams: When can we avoid interferences? MPRA Paper No. 32198.

[2] Alcantud, J.C.R., Garcìa-Sanz, M.D., 2010. Evaluations of infinite utility streams: Pareto-efficient and egalitarian axiomatics. MPRA Paper No. 20133.

[3] Asheim, G.B., 2010. Intergenerational equity. Annual Review of Economics, 2, 197-222.

[4] Asheim, G.B., Tungodden, B., 2004. Resolving distributional conflicts between generations. Economic Theory, 24, 221-230.

[5] Asheim, G.B., d'Aspremont, C., Banerjee, K., 2010. Generalized timeinvariant overtaking. Journal of Mathematical Economics, 46, 519-533.

[6] Basu, K., Mitra, T., 2003. Aggregating infinite utility streams with intergenerational equity: the impossibility of being Paretian. Econometrica, 71, 1557-1563. 
[7] Bossert, W., Sprumont, Y., Suzumura, K., 2007. Ordering infinite utility streams. Journal of Economic Theory, 135, 179-189.

[8] Crespo, J., Nuñez, C., Rincón-Zapatero, J., 2009. On the impossibility of representing infinite utility streams. Economic Theory, 40, 47-56.

[9] Diamond, P., 1965. The Evaluation of Infinite Utility Streams. Econometrica, 33, 170-177.

[10] Hara, C., Shinotsuka, T., Suzumura, K., Xu, Y., 2008. Continuity and egalitarianism in the evaluation of infinite utility streams. Social Choice and Welfare, 31, 179-191.

[11] Hammond, P., 1976. Equity, Arrow's Conditions and Rawls' Difference Principle. Econometrica, 44, 793-804.

[12] Lauwers, L., 1997. Rawlsian equity and generalised utilitarianism with an infinite population. Economic Theory, 9, 143-150.

[13] Lauwers, L., 2010. Ordering infinite utility streams comes at the cost of a non-Ramsey set. Journal of Mathematical Economics, 46, 32-37.

[14] Lombardi, M., Veneziani, R., 2009. Liberal Egalitarianism and the Harm Principle. WP No. 949, Queen Mary University of London.

[15] Mariotti, M., Veneziani, R., 2009a. Non-Interference Implies Equality. Social Choice and Welfare, 32, 123-128.

[16] Mariotti, M., Veneziani, R. 2009b. The paradoxes of the liberal ethics of non-interference. WP No. 953, Queen Mary University of London.

[17] Mariotti, M., Veneziani, R., 2010. On the impossibility of complete non-interference in Paretian social judgements. Mimeo, Queen Mary University of London.

[18] Zame, W.R., 2007. Can Intergenerational Equity be Operationalized? Theoretical Economics, 2, 187-202. 


\section{Addendum to "Liberal Principles for Social Wel- fare Relations in Infinitely-Lived Societies"}

\subsection{Independence of the axioms in Theorem 2}

For an example violating only $\mathbf{F A}$, define $\succcurlyeq$ on $X$ in the following way: $\forall_{1} u,{ }_{1} v \in X$

$$
\begin{aligned}
{ }_{1} u & ={ }_{1} v \Rightarrow{ }_{1} u \sim{ }_{1} v \\
\exists T & \in \mathbb{N}: u_{t}=v_{t} \forall t<T \text { and } u_{T}>v_{T} \Rightarrow{ }_{1} u \succ{ }_{1} v
\end{aligned}
$$

The SWR $\succcurlyeq$ on $X$ is not an extension of the leximax SWR $\succcurlyeq^{L X}$. The $\mathrm{SWR} \succcurlyeq$ on $X$ satisfies all axioms except FA.

For an example violating only SPO, define $\succcurlyeq$ on $X$ in the following way: $\forall_{1} u,{ }_{1} v \in X,{ }_{1} u \sim{ }_{1} v$. The SWR $\succcurlyeq$ on $X$ is not an extension of the leximax SWR $\succcurlyeq^{L X}$. Clearly, the swr $\succcurlyeq$ on $X$ satisfies all axioms except SPO.

For an example violating only IBP, let $\succcurlyeq$ on $X$ be the leximin swr (see Definition 1, p. 3). It follows that the swr $\succcurlyeq$ on $X$ is not an extension of the leximax SWR. The SWR $\succcurlyeq$ on $X$ satisfies all axioms except IBP.

For an example violating only $\mathbf{M C}$, define $\succcurlyeq$ on $X$ in the following way: $\forall{ }_{1} u,{ }_{1} v \in X$,

$$
\begin{aligned}
& \exists \pi \in \Pi:{ }_{1} u=\pi\left({ }_{1} v\right) \Rightarrow{ }_{1} u \sim{ }_{1} v, \\
& { }_{1} u>{ }_{1} v \Rightarrow{ }_{1} u \succ{ }_{1} v .
\end{aligned}
$$

The SWR $\succcurlyeq$ on $X$ is not an extension of the leximax SWR. The SWR $\succcurlyeq$ on $X$ satisfies all axioms except $\mathbf{M C}$.

For an example violating only $\mathbf{W P C}$, let $\succcurlyeq$ on $X$ be the leximax catching up defined in Section 5 above. It follows that the swR $\succcurlyeq$ on $X$ is not an extension of the leximax SWR. The SWR $\succcurlyeq$ on $X$ satisfies all axioms except WPC. [To see that WPC is violated let ${ }_{\text {con }} \epsilon$ denote the stream of constant 
level of well-being equal to $\epsilon \in \mathbb{R}$. Consider the following vectors: ${ }_{1} u=$ $\left(3,{ }_{\text {con }} 0\right)$ and ${ }_{1} v=\left(2,{ }_{\text {con }} 1\right)$. Then, $\left({ }_{1} u,{ }_{1} v\right) \notin \succcurlyeq$ and $\left(\left({ }_{1} u_{T},{ }_{T+1} v\right),{ }_{1} v\right) \in \succ_{T}^{L}$ $\forall T \in \mathbb{N}]$.

\subsection{Independence of the axioms in Theorem 4}

The proof that the axioms in Theorem 4 are tight immediately follows from the previous examples and from the characterisation of the set of ordering extensions of the leximin catching-up in Lombardi and Veneziani (2009, Theorem 5). 